\title{
Luminescence Decay of Colloidal Quantum Dots and Stretched Exponential (Kohlrausch) Relaxation Function
}

\author{
(C) E.N. Bodunov ${ }^{1}$, A.L. Simoes Gamboa ${ }^{2}$ \\ ${ }^{1}$ Department of Physics, Emperor Alexander I St. Petersburg State Transport University, \\ 190031 St. Petersburg, Russia \\ ${ }^{2}$ International Research and Education Centre for Physics of Nanostructures, ITMO University, \\ 197101 St. Petersburg, Russia \\ E-mail: evgeny.bodunov@inbox.ru
}

The room temperature non-exponential luminescence decay of colloidal quantum dots is investigated theoretically in an attempt to identify the underlying physical mechanisms responsible for the form of the decay. It is shown that a stretched exponential functional form of the luminescence decay can be understood in terms of long-range resonance energy transfer from quantum dots to acceptors (molecules, quantum dots, or anharmonic molecular vibrations) or by contact quenching by quenchers (surface traps, molecules or quantum dots) distributed statistically (Poisson distribution) on the surface of the dots. These non-radiative transition processes are assigned to different ranges of the stretching parameter $\beta$. 